\title{
Novel function of PITH domain-containing 1 as an activator of internal ribosomal entry site to enhance RUNX1 expression and promote megakaryocyte differentiation
}

\author{
Bin Lu $\cdot$ Xueqin Sun · Yuxuan Chen • Qi Jin • \\ Qin Liang • Shangqin Liu • Yamu Li • Yan Zhou • \\ Wenxin $\mathrm{Li} \cdot$ Zan Huang
}

Received: 22 June 2014 / Revised: 31 July 2014 / Accepted: 11 August 2014 / Published online: 19 August 2014

(C) Springer Basel 2014

\begin{abstract}
Introduction Altered gene expression coincides with leukemia development and may affect distinct features of leukemic cells. PITHDI was significantly downregulated in leukemia and upregulated upon PMA induction in K562 cells undergoing megakaryocyte differentiation. We aimed to study the function of PITHD1 in megakaryocyte differentiation.

Materials and methods K562 cells and fetal liver cells were used for either overexpression or downregulation of PITHD1 by retroviral or lentiviral transduction. FACS was used to detect the expression of CD41 and CD42 to measure megakaryocyte differentiation in these cells. Western blot and quantitative RT-PCR were used to measure gene expression. Dual luciferase assay was used to detect promoter or internal ribosomal entry site (IRES) activity.

Results Ectopic expression of PITHD1 promoted megakaryocyte differentiation and increased RUNX1 expression while PITHD1 knockdown showed an opposite phenotype. Furthermore, PITHD1 efficiently induced endogenous RUNX1 expression and restored megakaryocyte differentiation suppressed by a dominant negative
\end{abstract}

B. Lu, X. Sun authors contributed equally to this work.

Electronic supplementary material The online version of this article (doi:10.1007/s00018-014-1704-2) contains supplementary material, which is available to authorized users.

B. Lu · X. Sun · Y. Chen · Q. Jin · Y. Li · Y. Zhou - W. Li ·

Z. Huang $(\varangle)$

College of Life Sciences, Wuhan University, Wuhan 430072,

Hubei, People's Republic of China

e-mail: z-huang@whu.edu.cn

Q. Liang · S. Liu

Department of Hematology, Zhongnan Hospital of Wuhan

University, Wuhan, Hubei, People's Republic of China form of RUNX1. PITHD1 regulated RUNX1 expression at least through two distinct mechanisms: increasing transcription activity of proximal promoter and enhancing translation activity of an IRES element in exon 3. Finally, we confirmed the function of PITHD1 in regulating RUNX1 expression and megakaryopoiesis in mouse fetal liver cells.

Conclusion and significance PITHD1 was a novel activator of IRES and enhanced RUNX1 expression that subsequently promoted megakaryocyte differentiation. Our findings shed light on understanding the mechanisms underlying megakaryopoiesis or leukemogenesis.

Keywords PITHD1 - RUNX1 - IRES .

Myeloid leukemia $\cdot$ Megakaryocyte differentiation

\section{Introduction}

Leukemic cells lose their abilities to undergo terminal differentiation to produce functional mature blood cells and proliferate indefinitely. Forcing leukemic cells to undergo terminal differentiation has long been proposed to be a rational way to cure leukemia. Indeed, differentiation therapy is so successful in acute promyeloid leukemia (APL) that combinatory application of all-trans retinoic acid (ATRA) and arsenic trioxide (ATO) synergistically causes terminal differentiation of APL cells and achieves complete clinic remission in APL patients $[1,2]$. Recent study identified Aurora kinase A as a therapeutic target of MLN8237 to promote polyploidization and differentiation in acute megakaryoblastic leukemia (AMKL) [3]. Unfortunately, differentiation therapy is not well defined in other subtypes of myeloid leukemia possibly due to heterogeneous etiology with different molecular genetic 
aberrations. Thus, extensive study on the molecular basis of forced differentiation in leukemic cells may facilitate to develop rational anti-leukemia therapy.

Runt-related transcription factor 1 (RUNX1) plays an important role in normal hematopoiesis and its deficiency or dysfunction due to mutations and translocation is one of frequent genetic alterations in leukemia [4]. It is required for embryonic HSC but not for definitive HSC function. It is also essential for terminal differentiation of megakaryocytes at the polyploidization stage and for the maturation of $\mathrm{T}$ and $\mathrm{B}$ lymphocytes [5, 6]. Furthermore, RUNX1 negatively regulates the proliferation of HSCs and myeloid progenitors [7, 8]. In leukemia, the mutant RUXN1 or chimeric gene product repressed the function of remaining endogenous RUNX1 in hematopoiesis [4], suggesting that the expansion of hematopoietic stem and progenitor cells due to RUNX1-deficiency or dysfunction may be an important cause of human leukemias. Enhancing RUNX1 activity by upregulating the remaining endogenous RUNX1 expression may push leukemic cell differentiation.

Transcription of RUNX1 is initiated at two distinct promoters, designated as the distal promoter (P1) and the proximal promoter $(\mathrm{P} 2)$, which leads to the production of three isoforms of RUNX1a/b/c [9]. P1 drives transcription of RUNX1c and its 5'UTR directs conventional capdependent translation of RUNX1c. P2 drives transcription of RUNX1a/b and multiple ATG in $5^{\prime}$ UTR impairs capdependent translation of RUNX1a/b. Instead, P2-UTR contains an internal ribosome entry site (IRES) element that is critical for translation of RUNX1a/b [7]. P1-UTRdependent RUNX1 expression is not required for embryonic survival but affects hematopoietic development while P2-UTR-dependent RUNX1 expression is critical for definitive hematopoiesis, leukemogenesis, angiogenesis, thymic development, and postnatal survival [10]. These findings highlight the necessity of tight control of RUNX1 expression and the pivotal role of IRES element in RUNX1 regulation. Indeed, a previous study demonstrated that IRES-dependent translation of RUNX1 increased during megakaryocyte differentiation in K562 cells [11]. Thus, modulating RUNX1 expression through IRES-dependent mechanism is critical for RUNX1 function in hematopoiesis or leukemogenesis.

In an effort to identify genes that promotes leukemic cell differentiation, we noticed that a novel gene PITHD1 [PITH (C-terminal proteasome-interacting domain of thioredoxin-like) domain-containing 1] was significantly downregulated in leukemia but dramatically upregulated upon PMA treatment in K562 cells undergoing megakaryocyte differentiation. PITHD1 bears a PITH domain and belongs to thioredoxin (TRX) family based on conserved domain analysis (http://www.ncbi.nlm.nih.gov/Structure/ cdd/). Except that we previously identified PITHDI as a differential expression gene in human embryogenesis [12, 13], little information about its function is available. Here, we showed that PITHD1 promoted megakaryocytic differentiation in K562 and HEL cells as well as primary mouse fetal liver cells. Moreover, PITHD1 acted as an activator of internal ribosomal entry site (IRES) on exon 3 of RUNX1 gene to enhance the RUNX1 translation. Thus, our study has identified novel function of PITHD1 and may provide useful information for understanding megakaryopoiesis as well as developing novel anti-leukemia strategy.

\section{Materials and methods}

\section{Cell cultures and blood samples}

Human myeloid leukemia cell lines K562 and HEL were cultured in RPMI 1640 medium (Gibco BRL, Grand Island, NY, USA) and HEK293T cells were cultured in Dulbecco's modified Eagle medium (DMEM) (Gibco BRL, Grand Island, NY, USA) supplemented with $10 \%$ fetal bovine serum, streptomycin, and penicillin, at $37{ }^{\circ} \mathrm{C}$ under $5 \% \mathrm{CO}_{2}$. Peripheral blood samples were obtained from newly diagnosed myeloid leukemia patients and healthy donors. Mouse (ICR strain) primary megakaryocytes cultured from fetal liver cells and purification of mature megakaryocyte were performed as previously described $[14,15]$. Mononuclear cells (MNCs) were isolated from peripheral blood samples on Ficoll-Hypaque density gradients. All experiments involving in human blood samples were approved by the Medical Ethics Committee of Zhongnan Hospital of Wuhan University. Consent form was obtained from each patient and healthy donor. Animal studies were approved by the Animal Care and Use Committees of Wuhan University College of Life Sciences.

Lentivirus or retrovirus infection

PITHD1 overexpression and knockdown cell lines were achieved through lentiviral or retroviral transduction as previously described [16]. Lentiviral vector pHAGE or retroviral vector pMSCVpuro was used for overexpression and lentiviral vector pLKO.1 was used for knockdown. Vectors carried either puromycin resistant gene or GFP for selection as indicated. Short hairpin RNA sequences for knocking down PITHD1 were determined by online shRNA searching tool (http://www.sirnawizard.com/design advanced.php), and blast sequences at NCBI to avoid offtarget. The sequences of shRNA oligos specific for human PITHD1 used in this study were as following: shRNA\#1: GGGAGAGGATGATGACTCACA; shRNA\#2: GATGAGACTGTACAAGAATAT. The sequences of shRNA oligos specific for mouse Pithdl were as following: shRNA/ 
m\#1: GACGAAGAGCTCCTGTTTAAT; shRNA/m\#2: GATGAGACTGTACAAGAACAT. Empty plasmids were used as controls.

\section{Flow cytometry analysis}

K562 or HEL cells were incubated with PMA (10 nM) for hours as indicated. The treated cells were washed with PBS and stained with phycoerythrin (PE)-conjugated anti-CD61 antibody or anti-CD41 antibody (BD Biosciences, San Jose, CA, USA). CD61 or CD41 expression was measured by flow cytometry on FACSCalibur (BD Biosciences, San Jose, CA, USA) and analyzed with FlowJo software (TreeStar, Ashland, OR). All flow cytometry data were the representative results from three independent experiments with duplicates.

\section{Quantitative RT-PCR analysis}

Total RNA was extracted from cells using TRIzol reagent (Invitrogen, Grand Island, NY, USA), and reverse-transcribed into cDNA using High-Capacity Reverse Transcription Kit (Applied Biosystems, Grand Island, NY, USA). Quantitative RT-PCR was performed as following conditions: $95{ }^{\circ} \mathrm{C}$ for $15 \mathrm{~min}$ followed by $95^{\circ} \mathrm{C}$ for $30 \mathrm{~s}$, $60{ }^{\circ} \mathrm{C}$ for $30 \mathrm{~s}, 72{ }^{\circ} \mathrm{C} 1 \mathrm{~min}$ for 40 cycles. The reactions were run in triplicate on the $7900 \mathrm{HT}$ Fast Real-Time PCR System (Applied Biosystems, Grand Island, NY, USA). The relative quantitation of real-time PCR product was measured using the comparative $\Delta \Delta \mathrm{CT}$ method. All quantitative RT-PCR data were the representative results from three independent experiments with duplicates. Sequences of primer sets used for quantitative RT-PCR are available upon request.

\section{Immunoblotting and antibodies}

Immunoblotting was performed as previously described [14]. Antibodies used in this study include goat antiGATA-1, rabbit anti-FLI1, mouse anti-RUNX1, mouse anti-HSC70 (Santa Cruz Biotechnology, Santa Cruz, CA, USA); rabbit anti-phospho-ERK, anti-phospho-JNK, antiphospho-p38, anti-phospho-NF-кB (Cell Signaling Tech); mouse anti-GAPDH, anti-lamin A/C, anti-HA (ProteinTech Group, Chicago, IL, USA); mouse anti-Flag (Sigma, St Louis, MO, USA). HSC70, GAPDH, Actin, and lamin $\mathrm{A} / \mathrm{C}$ antibodies served as loading controls. Goat antimouse, donkey anti-goat, and goat anti-rabbit IgG horseradish peroxidase (HRP)-conjugated were purchased from Jackson ImmunoResearch. PITHD1 rabbit polyclonal antibody was raised against full-length protein of human PITHD1 expressed in bacteria (BL21). All immunoblots were the representative data from three independent experiments with similar results.
Nucleus and cytoplasm extraction

Briefly, 5 to $10 \times 10^{6}$ cells were collected and incubated in cytoplasm lysis buffer (20 mM HEPES pH 7.4, $10 \mathrm{mM}$ $\mathrm{KCl}, 1 \mathrm{mM}$ EDTA, $10 \%$ glycerol) with $0.2 \% \mathrm{NP}-40$ for $10 \mathrm{~min}$ on ice in the presence of protease inhibitors. Cell lysates were centrifuged at 14,000 rpm for $10 \mathrm{~min}$, and the supernatant was collected as cytoplasm extraction. The pellets were washed once and resuspended in nucleus lysis buffer (20 mM HEPES pH 7.4, $10 \mathrm{mM} \mathrm{KCl,} 1$ mM EDTA, $20 \%$ glycerol, $350 \mathrm{mM} \mathrm{NaCl}$, protease inhibitors), and nuclear extraction was harvested after centrifugation (16,000 g, $10 \mathrm{~min})$.

Luciferase assay

K562 cells were transfected with RUNX1 IRES reporter vector together with empty control or Flag-PITHD1. The luciferase activity was assayed with a Dual-Luciferase Reporter Assay System following the manufacturer's instructions (Promega, Madison, WI, USA). The RUNX1 IRES activity was presented as relative luciferase activity by normalizing the firefly luciferase activity to renila luciferase activity. For RUNX1 P2 promoter activity, an internal control of renila luciferase expressing vector was used for transfection of K562 cells together with RUNX1 P2 promoter reporter vector in combination with empty control or Flag-PITHD1 expressing vector. Data were representative results from three independent experiments with similar results.

\section{Statistics}

All statistical analyses were performed using the Student's $t$ test (2 tailed, unpaired). A $p$ value of 0.05 or less was considered as significance.

\section{Results}

PITHD1 is downregulated in leukemia and upregulated upon forced differentiation in leukemic cells

Previously, differential expression pattern of PITHD1 was observed in human embryo development and PITHD1 was predicted to function in hematopoiesis. To probe its potential function, we first detected PITHD1 expression in hematopoietic cell lines. We found that PITHD1 was relatively high in G1ME cells, an immortalized cell line derived from mouse embryo stem cells, and mononuclear cells (MNCs) isolated from peripheral blood of healthy donor. In contrast, PITHD1 expression was rather low in all malignant leukemic cell lines examined including 
A
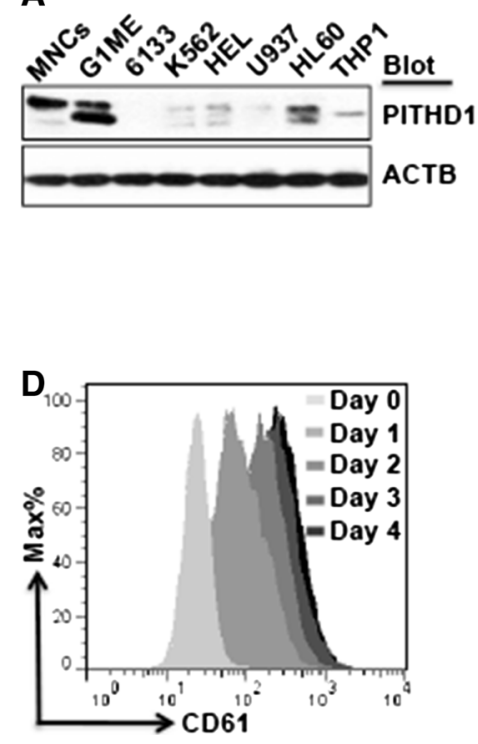
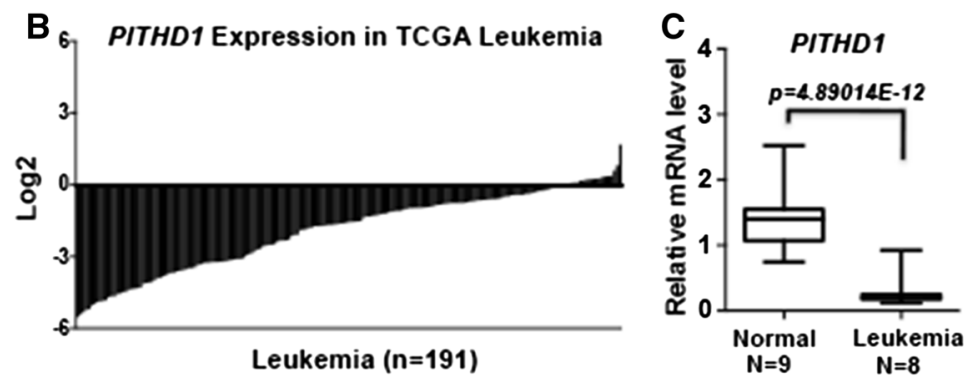

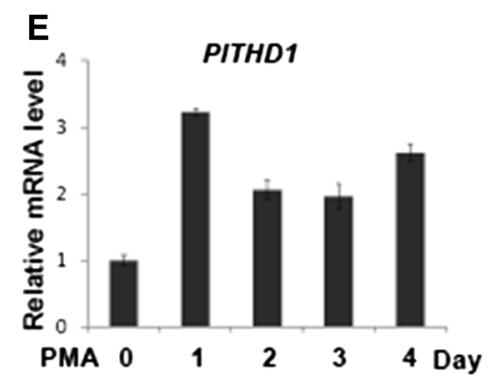

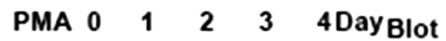

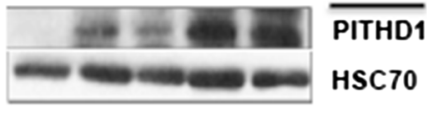

Fig. 1 PITHD1 is downregulated in leukemia and upregulated upon forced differentiation in megakaryocytes. a The expression of PITHD1 protein in cell lines and mononuclear cells (MNCs) from healthy donor was measured by western blot. b The expression of PITHD1 mRNA in leukemia $(n=191)$ was analyzed and presented as $\log 2$ median-centered intensity. Expression data were obtained from the TCGA database (http://cancergenome.nih.gov/). c Quantitative RT-PCR analysis of PITHD1 in MNCs from healthy volunteer donors (Normal, $N=9$ ) and acute myeloid leukemia patients (AML, $N=8$ ). The expression of PITHD1 was normalized to GAPDH and

presented as relative mRNA level. d K562 cells treated with PMA for days as indicated. The megakaryocyte differentiation of K562 cells was assayed by measuring CD61 expression through staining cells with PE-conjugated anti-CD61 antibody and analyzed by flow cytometry. Histogram was representative data from three independent experiments (duplicates) with similar results. e The PMA-treated K562 cells were harvested to measure the mRNA (left panel) and protein (right panel) expression level of PITHD1 by quantitative RTPCR and western blot, respectively. GAPDH and HSC70 served as controls, respectively

6133, K562, HEL, HL-60, U937, and THP1 (Fig. 1a). These observations suggest a correlation in PITHD1 expression and leukemic cells. Therefore, we investigated the online microarray database of human leukemia samples and found that PITHDI was dramatically downregulated in these samples $(N=191)$ (Fig. 1b). Furthermore, we measured PITHDI expression in primary acute myeloid leukemia (AML) cells freshly isolated from patients. Noticeably, PITHD1 expression was significantly downregulated in AML compared to that of healthy control (Fig. 1c). These observations suggest that PITHD1 may play a role in normal blood cell differentiation and its downregulation correlates to leukemia. In supporting this idea, PITHD1 upregulation at both mRNA and protein level was observed in K562 cells undergoing megakaryocyte differentiation induced by PMA (Fig. 1d, e). PITHD1 upregulation seemed not a special case in PMA-induced megakaryocyte differentiation in K562 cells. Similar phenotype was observed in HEL cells treated with PMA (Figure S1A). In addition, PITHD1 was also efficiently upregulated in K562 cells induced by cytosine arabinoside (Ara-C) or hemin (Fig. 2a, c). These results suggest that PITHD1 upregulation correlates to leukemic cell differentiation.
PITHD1 enhances PMA-induced megakaryocyte differentiation in K562 cells

Based on the correlation between PITHD1 upregulation and leukemic cell differentiation, we speculated that PITHD1 might contribute to forced leukemic cell differentiation. To explore the potential role of PITHD1, we chose PMA-induced megakaryocyte differentiation in K562 cells as a cell model. We overexpressed PITHD1 through lentiviral transduction and confirmed its overexpression by quantitative RT-PCR and western blot (Fig. 2a, b). Upon PMA treatment for $48 \mathrm{~h}$, ectopic expression of PITHD1 significantly enhanced megakaryocyte differentiation compared to control cells, evidenced by increased expression level of surface markers CD61 and CD41 specific for megakaryocytes (Fig. 2c, d). These observations suggest that PITHD1 may enhance PMA-induced megakaryocyte differentiation in K562 cells. Consistent to this idea, HEL cells overexpressing PITHD1 also showed more effective megakaryocyte differentiation induced by PMA compared to control cells (Figure S1b, c). To further confirm the function of PITHD1, we knocked down PITHD1 in K562 cells by lentivirus-mediated shRNA technique. The efficiency of PITHD1 knockdown by two short hairpin 

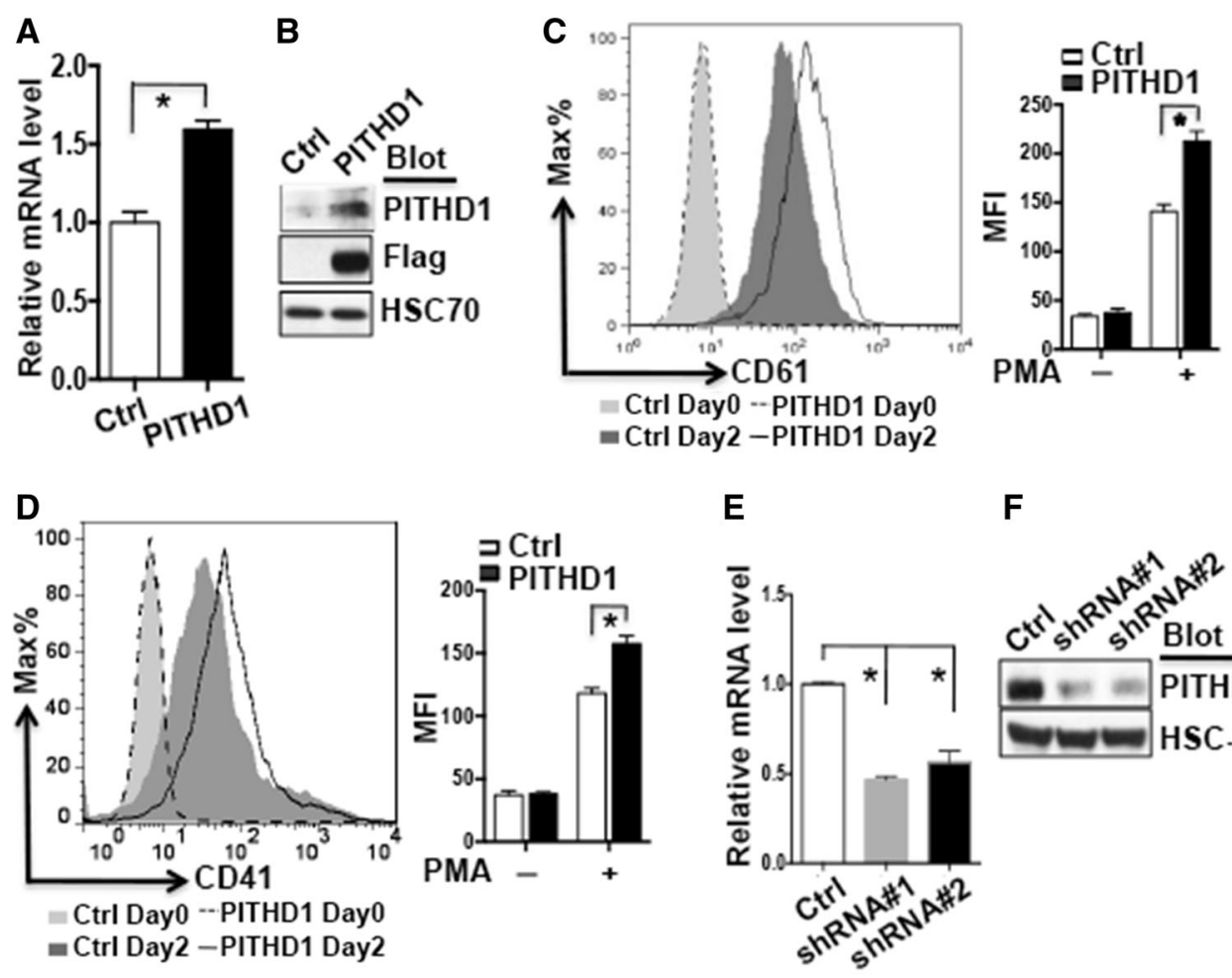

$\mathbf{F}$
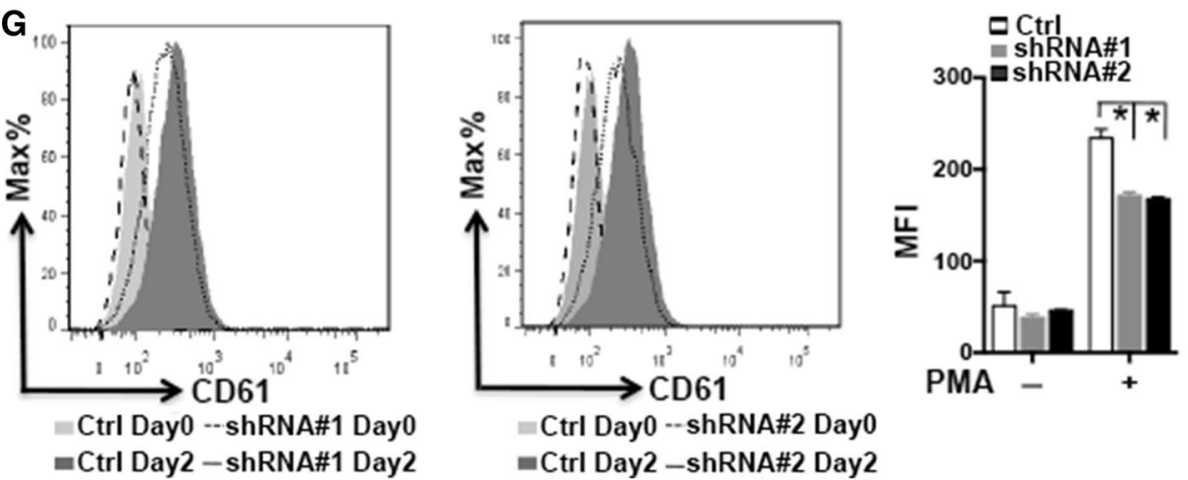

Fig. 2 PITHD1 promotes PMA-induced megakaryocytic differentiation. a K562 cells were transduced with control lentiviral vector (Ctrl) or a PITHD1-expressing lentiviral vector. The mRNA expression level of PITHD1 in the resultant cells was confirmed by quantitative RT-PCR and presented as relative mRNA level. b The endogenous or exogenous PITHD1 protein in the resultant cells was detected by western blot using antibody specific for PITHD1 or antibody specific to Flag tag fused to exogenous PITHD1. HSC70 served as a loading control. c The resultant cells were cultured with PMA for 2 days (Ctrl Day 2 and PITHD1 Day 2) or without PMA (Ctrl Day 0 and PITHD1 Day 0). The CD61 expression was measured by staining cells with PE-conjugated anti-CD61 antibody and analyzed by flow cytometry (histogram, left panel). Bar graph (right panel) is the statistics of mean fluorescence intensity (MFI) of flow cytometry analysis. d The expression of CD41 in the resultant cells was also measured and analyzed (histogram, left panel). Bar graph

RNAs (shRNA\#1, \#2) was confirmed by quantitative RTPCR and western blot (Fig. 2e, f). As expected, PITHD1 knockdown impaired PMA-induced megakaryocyte (right panel) is the statistics of mean fluorescence intensity (MFI) of flow cytometry analysis. e K562 cells were transduced with control lentiviral vector (Ctrl) or lentiviral vectors expressing two shRNAs specific for human PITHD1 (shRNA\#1, shRNA\#2). The endogenous mRNA level of PITHD1 in resultant cells was detected by quantitative RT-PCR. $\mathbf{f}$ The downregulation of endogenous PITHD1 protein was confirmed by western blot with specific antibody against PITHD1. g The PITHD1-downregulated cells (shRNA\#1 and shRNA\#2) and control cells (Ctrl) were cultured with PMA for 2 days. The expression of CD61 in the resultant cells was detected (histogram, left panel). Bar graph (right panel) is the statistics of mean fluorescence intensity (MFI) of flow cytometry analysis. All histograms were representative results of three independent experiments (duplicates) with similar results. *Indicates significance $(p<0.05)$

differentiation with reduced CD61 expression (Fig. 2g). Taken together, these findings suggest that PITHD1 enhances megakaryocyte differentiation. 


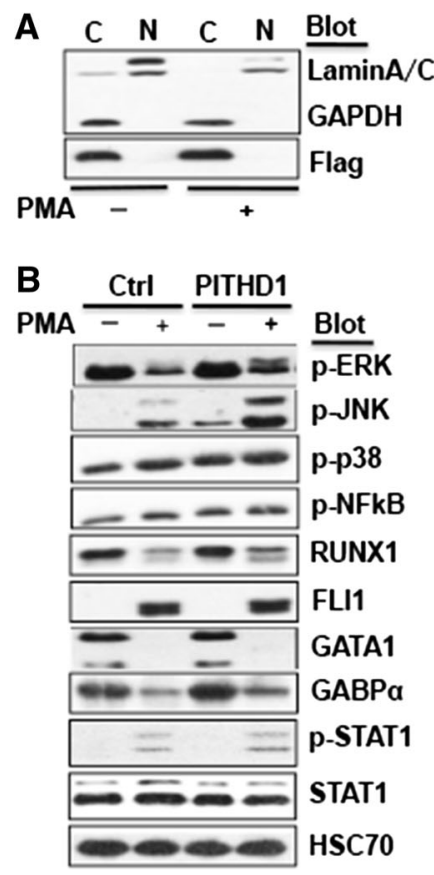

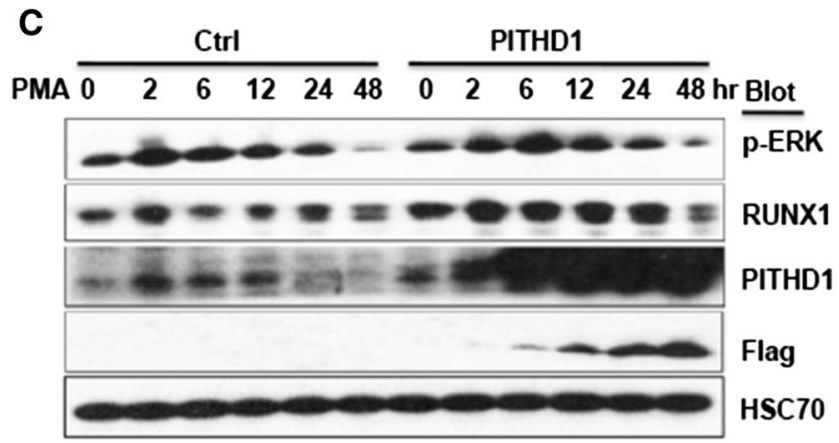

D

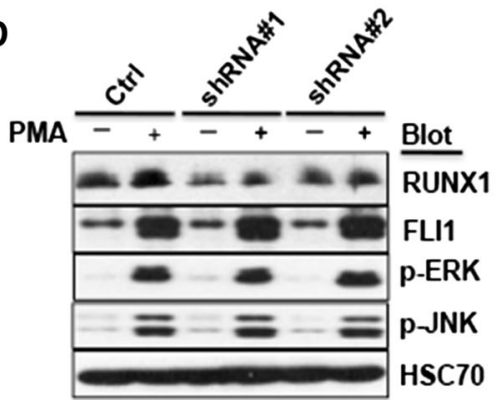

Fig. 3 PITHD1 up-regulates RUNX1 protein during megakaryocyte differentiation. a K562 cells overexpressing PITHD1 were treated with PMA (+) or without (-) for 2 day. Cytoplasmic extracts (C) or nucleus extracts $(\mathrm{N})$ were fractionated from the resultant cells and subjected to detect Flag-tagged PITHD1 by western blot. GAPDH and Lamin A/C served as controls, respectively. b K562 cells transduced with control (Ctrl) or PITHD1-overexpressing lentiviral vector were induced with $(+)$ or without $(-)$ PMA for 2 days. The phosphorylation of ERK (p-ERK), JNK (p-JNK), P38 (p-P38), and NFKB (pNFkB), the expression of RUNX1, FLI1, GATA1 and GABP $\alpha$ were

PITHD1 enhances PMA-induced RUNX1 upregulation during megakaryocyte differentiation

As a novel gene, little information on PITHD1 is available. To explore potential mechanism by which PITHD1 enhanced PMA-induced megakaryocyte differentiation, we first examined the subcellular distribution of PITHD1. Apparently, PITHD1 was only detected in the cytoplasm extract (C) but not in nuclear extract $(\mathrm{N})$ and PMA treatment did not alter the distribution pattern (Fig. 3a). Thus, it seemed likely that PITHD1 might act as a signaling molecule. PMA treatment primarily activates several downstream signaling pathways that promoted megakaryocyte differentiation [17]. To test whether PITHD1 affects PMA-induced signaling in the cytoplasm, we measured the activation of several known downstream signaling molecules including ERK, JNK, p38 and NK-кB. PITHD1 increased the phosphorylation of ERK and JNK but did not significantly affect the phosphorylation of $\mathrm{p} 38$ or NK-kB (Fig. 3b, c). Phosphorylation of STAT1, a known signaling molecule responsible for megakaryocyte differentiation, was also increased upon PMA stimulation analyzed by western blot. HSC70 served as loading control. c Control (Ctrl) or PITHD1-expressing K562 cells (PITHD1) were treated with PMA for indicated hours and were subjected to western blot to detect phosphorylation of ERK (p-ERK) and the expression of RUNX1 and PITHD1 proteins. HSC70 served as loading control. d Control (Ctrl) or PITHD1 knockdown (shRNA\#1 and shRNA\#2) K562 cells were treated with PMA for $24 \mathrm{~h}$ and harvested to detect the phosphorylation of ERK (p-ERK) and JNK (p-JNK) and the expression of RUNX1 and FLI1

and PITHD1 did not affect PMA-induced STAT1 phosphorylation. In addition, we measured the expression of several transcription factors that played important roles in megakaryocyte differentiation [18-20]. We discovered that PITHD1 overexpression increased RUNX1 protein but not for GATA1, FLI1, or GABP $\alpha$ (Fig. 3b, c). Previously, JNK was shown to upregulate FLI1 and ERK activated RUNX1 [14]. However, we failed to see increased FLI1 in our experiment setting. What is more, RUNX1 upregulation in response to PMA treatment was significantly reduced when PITHD1 was knocked down. In contrast, PITHD1 downregulation did not significantly affect ERK and JNK phosphorylation (Fig. 3d). Therefore, it was very likely that PITHD1 enhanced PMA-induced megakaryocyte differentiation by increasing RUNX1 expression.

PITHD1 enhances PMA-induced megakaryocyte differentiation by increasing RUNX1 expression

ERK activation is known to activate RUNX1 [5, 14]. To further evaluate the contribution of ERK activation in PITHD1-enhanced megakaryocyte differentiation, we 
A

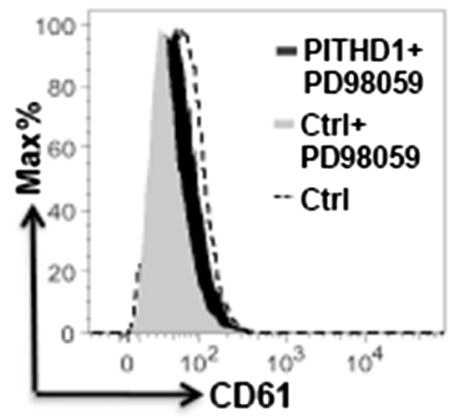

C

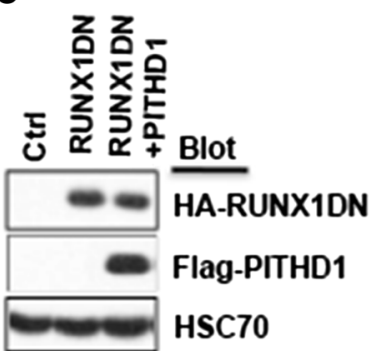

E
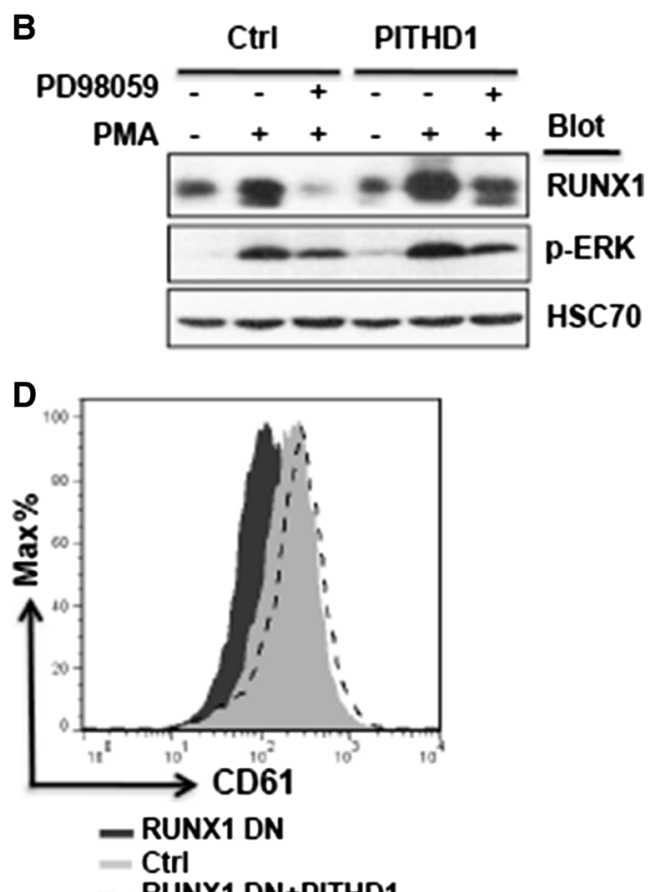

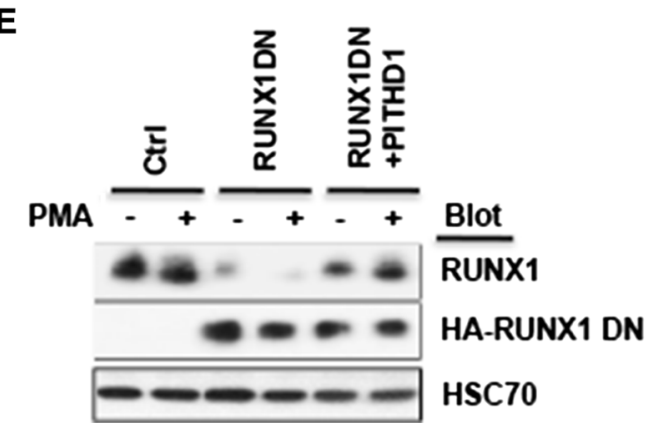

Fig. 4 Up-regulation of RUNX1 is essential for the function of PITHD1 in megakaryocytic differentiation. a K562 cells transduced with control lentiviral vector $(\mathrm{Ctrl})$ or PITHD1-expressing lentiviral vector (PITHD1) were pretreated with or without PD98059 and induced with PMA for 2 days. Cells were stained with PE-conjugated anti-CD61 antibody and analyzed by flow cytometry. Histogram was representative data from three independent experiments (duplicates) with similar results. b Cells above were also harvested to detect the phosphorylation of ERK (p-ERK) and expression of RUNX1. HSC70 served as loading control. c K562 cells were transduced with pHAGE and MSCV lentiviral vector (Ctrl), pHAGE and dominant-negative

RUNX1-expressing (RUNX1DN) lentiviral vectors, PITHD1 and RUNX1DN expressing lentiviral vectors (PITHD1+RUNX1DN). HA-tagged RUNX1DN and Flag-tagged PITHD1 in the resultant cells were detected by western blot. HSC70 served as loading control. d The resultant cells treated with PMA for 2 days were harvested to stain with PE-conjugated anti-CD61 antibody and analyzed by flow cytometry. Histogram was representative data from three independent experiments (duplicates) with similar results. e The resultant cells treated with $(+)$ or without $(-)$ PMA were analyzed by western blot. The endogenous RUNX1 and HA-tagged RUNX1DN were detected. HSC70 served as loading control

treated cells with PD98059, a specific MEK1/2 inhibitor. As expected, PD98059 treatment (Ctrl+PD98059) suppressed PMA-induced megakaryocyte differentiation with reduced CD61 expression compared to control cells (Ctrl) (Fig. 4a). PD98059 also efficiently inhibited PMA-induced ERK phosphorylation and reduced PMA-induced upregulation of RUNX1 (Fig. 4b). Furthermore, PITHD1 overexpression (PITHD1+PD98059) partially rescued megakaryocyte differentiation inhibited by PD98059 compared to control cells treated with PD98059 (Ctrl+PD98059) (Fig. 4a). PITHD1 marginally increased

PMA-induced ERK phosphorylation and exerted more pronounced effect on RUNX1 upregulation (Fig. 4b). Interestingly, PITHD1 failed to rescue ERK phosphorylation but robustly restored RUNX1 upregulation suppressed by PD98059 treatment (Fig. 4b). These findings suggest that PITHD1 may enhance PMA-induced megakaryocyte differentiation mainly by augmenting RUNX1 expression. Our data also suggest that PITHD1 may in part upregulate RUNX1 through an ERK-independent mechanism.

To verify that PITHD1 enhanced PMA-induced megakaryocyte differentiation by increasing RUNX1 
expression, we repressed endogenous RUNX1 by overexpressing a dominant negative form of RUNX1 (RUNX1 DN) (Fig. 4c) [21]. As expected, RUNX1 DN significantly inhibited endogenous RUNX1 expression and suppressed PMA-induced megakaryocyte differentiation with reduced CD61 expression (Fig. 4d, e). Noticeably, PITHD1 effectively antagonized the inhibitory effect of RUNX1 DN on megakaryocyte differentiation as PITHD1 significantly restored CD61 expression in RUNX1 DN overexpression cells comparable to that of control cells (Fig. 4d). PITHD1 also efficiently rescued the expression of endogenous RUNX1 expression compared to control cells (Fig. 4e). These findings suggest that PITHD1 enhances PMAinduced megakaryocyte differentiation in part by increasing RUNX1 expression.

PITHD1 enhances translation activity of IRES element

Previous studies demonstrated that RUNX1 expression was regulated at both transcription and translation levels. To probe the mechanism by which PITHD1 regulates RUNX1 expression, we first measured mRNA expression of $R U N X 1 a / b$. We found that PMA treatment significantly upregulated $R U N X 1 a / b$ mRNA. Noticeably, PITHD1 further enhanced PMA-induced upregulation of RUNXIa/b mRNA. In contrast, downregulation of PITHDI (shRNA\#1, shRNA\#2) showed an opposite phenotype (Fig. 5a, b). IRES element on the exon 3 mediated stability of $R U N X 1 a / b$ in the presence of PMA and contributed to RUNX1 upregulation [11]. To address whether PITHD1 increased RUNX1 mRNA by enhancing transcription, we cloned P2 promoter of RUNX1 without the IRES element on the exon 3 into a luciferase reporter vector and performed luciferase activity assay in K562 cells. Consistently, PITHD1 increased luciferase activity in the presence of PMA treatment suggesting that PITHD1 may enhance P2 promoter transcription activity (Fig. 5c). Therefore, it is very likely that PITHD1 increases RUNX1 mRNA in part by activating $\mathrm{P} 2$ promoter of RUNX1 gene.

Previous study also demonstrated that the IRES element on the $5^{\prime}$ UTR of $R U N X 1 a / b$ mRNA enhanced the stability and translation of $R U N X 1$ mRNA that contributed to upregulation of RUNX1 during megakaryocyte differentiation [11]. To further address whether the IRES element mediated the increased RUNX1 protein by PITHD1, we constructed an IRES reporter vector (Fig. 5d, top panel). Since renila luciferase (Rluc) and firefly luciferase (Fluc) were expressed as one single mRNA molecule, increased relative luciferase activity (Fluc normalized by Rluc) would reflect the increased IRES-dependent translation of firefly luciferase. As expected, PMA treatment increased the relative luciferase activity up to two folds in $24 \mathrm{~h}$ in K562 cells, suggesting an increase of the translation activity of RUNX1 IRES. More importantly, PITHD1 further increased the relative luciferase activity more than three folds (Fig. 5d). In contrast, PITHD1 failed to do so when the RUNX1 IRES element was replaced by a HSVTK promoter (Figure S3A). Thus, these findings strongly suggest that PITHD1 is involved in IRES-dependent translation control of RUNX1 during megakaryocyte differentiation. To further confirm that PITHD1 universally regulates IRES-mediating protein translation, we tested the effect of PITHD1 one encephalomyocarditis virus (ECMV) IRES-mediated protein translation that widely used for researches. As shown in Fig. 5e, GATA1 and GFP were expressed in one mRNA molecule and GFP translation was dependent on IRES. PITHD1 significantly increased GFP protein when GATA1 was used for normalization. In addition, PITHD1 failed to alter GFP expression when the IRES sequence was substituted by a PGK promoter (Figure S3B). These findings highlight the pivotal role of PITHD1 in regulating IRES-dependent translation.

PITHD1 promotes megakaryocyte differentiation in mouse fetal liver cells

The IRES element in the Runxl gene is required for angiogenesis, hematopoiesis and leukemogenesis in Runxl knockin mice deleted for IRES element [10]. To demonstrate the role of PITHD1 in megakaryopoiesis, mouse fetal liver cells were transduced with control lentiviral vector (Ctrl) or PITHD1-overexpressing (PITHD1) vector for megakaryocyte culture. PITHD1 overexpression significantly enhanced megakaryocyte differentiation compared to control cells, evidenced by increased expression level of surface markers CD41 and CD42 specific for megakaryocytes (Fig. 6a, b). Consistently, forced expression of PITHD1 also upregulated the expression of Runxla/b in megakaryocytes (Fig. 6c). Further supporting the function of PITHD1 in promoting megakaryopoiesis, mouse fetal liver cells transduced with lentiviral vectors expressing two individual shRNAs specific for mouse Pithdl (shRNA/ $\mathrm{m \# 1}$, shRNA/m\#2) showed impaired megakaryopoiesis with reduced expression of megakaryocyte-specific surface antigens CD41 and CD42 (Fig. 6d, e). As expected, the Runxl expression in Pithdl knockdown megakaryocytes was consequently reduced (Fig. 6f). These findings strongly support the idea that PITHD1 may promote megakaryopoiesis in part by upregulating RUNX1 expression under physiological conditions.

\section{Discussion}

PITHD1 belongs to TRX family based on conserved domain analysis. TRX family contains an active dithiol site 

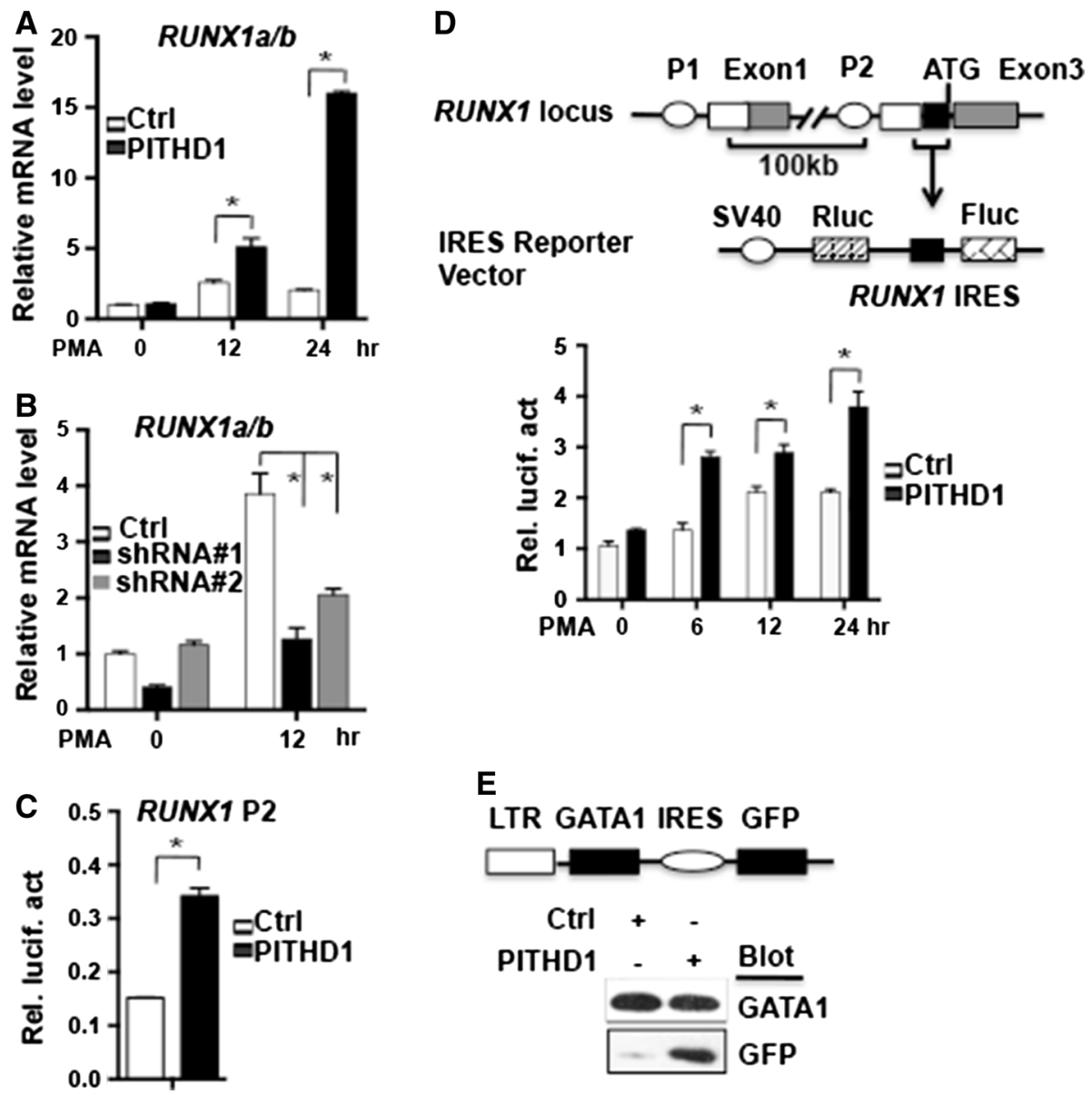

E

LTR GATA1 IRES GFP

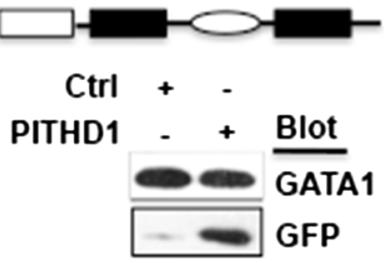

Fig. 5 PITHD1 regulates IRES-dependent translation of RUNX1. a Control (Ctrl) or PITHD1 overexpression (PITHD1) K562 cells were treated with PMA for hours as indicated. Cells were harvested and the RUNX1a/b mRNA was measured by quantitative RT-PCR and presented as relative mRNA level. b Control (Ctrl) or PITHD1 knockdown K562 cells (shRNA\#1 and shRNA\#2) were treated with PMA for times as indicated. Cells were harvested and the RUNX1a/b mRNA was measured by quantitative RT-PCR and presented as relative mRNA level. c K562 cells were transfected with RUNX1 P2 reporter vector along with control vector (Ctrl) or PITHD1-expressing vector (PITHD1). The resultant cells were treated with PMA for $12 \mathrm{~h}$ and luciferase activity was analyzed. d Schematic illustration of RUNX1 gene locus and the IRES reporter vector (top). K562 cells

presented in a CXXC motif and reduces protein disulfide bonds, resulting in a disulfide bond at its active site. By altering their redox state, TRX regulates the functions of at least 30 target proteins. It also plays an important role in the defense against oxidative stress [22]. To address whether PITHD1 function as a potential thioredoxin, we measured ROS product in PITHD1 overexpressing K562 cells. PITDH1 did not significantly reduce ROS product induced by PMA treatment, consistent to its lack of CXXC motif as critical active site for thioredoxin (data not shown). These observations suggest that PITHD1 may not function as a typical thioredoxin. In fact, our findings demonstrated that PITHD1 functioned to regulate

were transfected with RUNX1 IRES reporter vector in combination with control vector (Ctrl) or PITHD1-expressing vector (PITHD1). Cells were treated with PMA for indicated hours and the dual luciferase activity was measured (bottom bar graph). The RUNX1 IRES activity was presented as relative luciferase activity by normalizing the firefly luciferase activity to renila luciferase activity. e Schematic illustration of a vector expressing GATA1 and GFP bicistronically (top). 293T cells were transfected with GATA1/GFP expression vector together with control vector (Ctrl) or PITHD1expressing vector (PITHD1). Cells were harvested and the expression of GATA1 and GFP was detected by western blot. GATA1 expression served as control. *Indicates significance $(p<0.05)$

megakaryocyte differentiation by modulating RUNX1 expression. RUNX1 deficiency due to point mutation or translocation is one of most important genetic events leading to leukemia [4]. PITHD1 seemed to upregulate endogenous RUNX1 and antagonize the effect of dominant negative form of RUNX1, which mimics the inhibitory effect of RUNX1 genetic alterations found in leukemia, on PMA-induced megakaryocyte differentiation.

Although the function of RUNX1 in embryo hematopoiesis and leukemogenesis has been well established, the regulation of RUNX1 expression has not been well addressed. Particularly, an IRES-dependent regulation of RUNX1 expression seems critical for RUNX1 function 
A
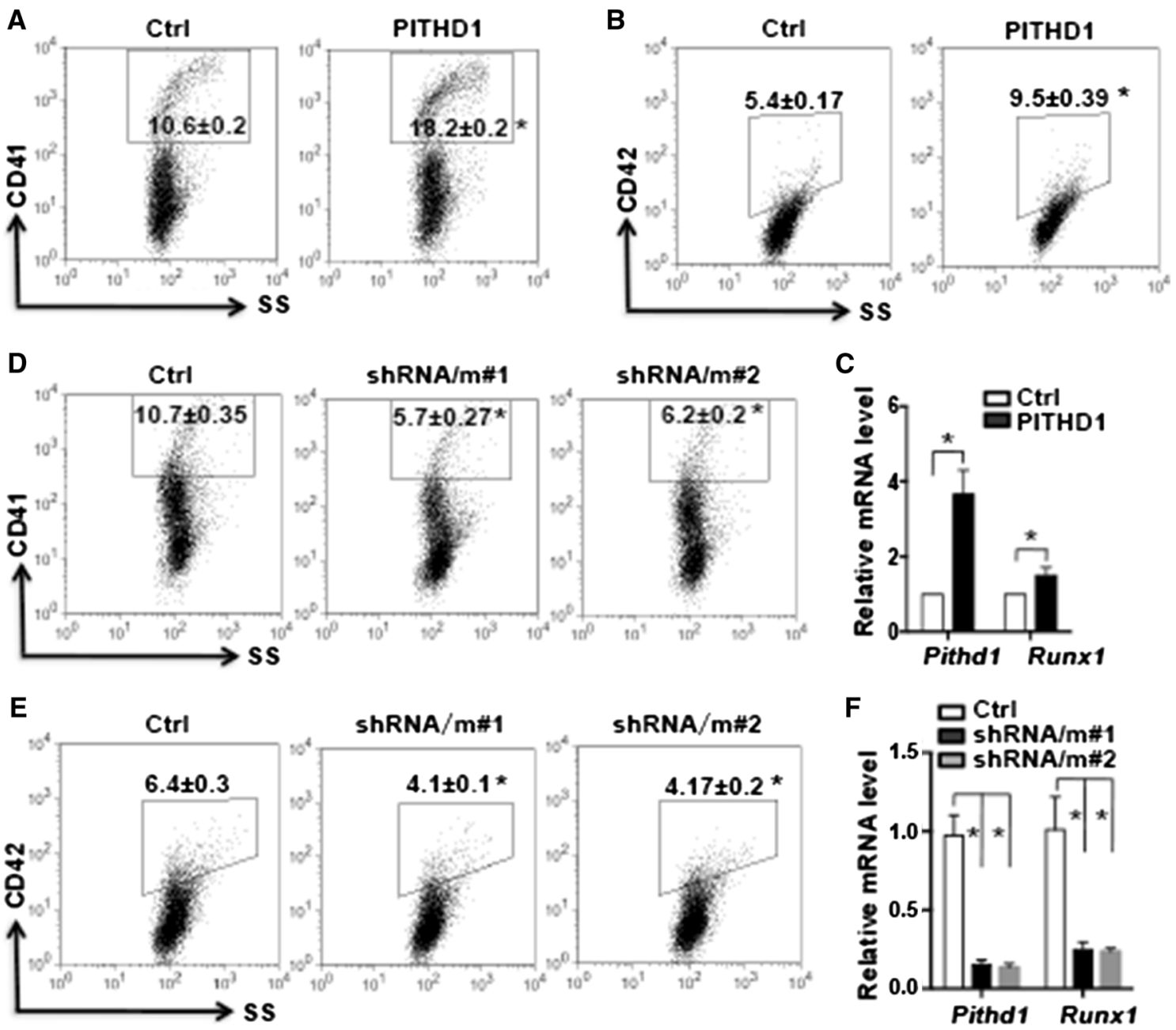

Fig. 6 PITHD1 promotes megakaryocyte differentiation in mouse fetal liver cells. a Mouse fetal liver cells were transduced with control lentiviral vector (Ctrl) or PITHD1-overexpressing (PITHD1) vector and cultured for megakaryocytes. The resultant cells were stained with PE-labeled anti-CD41 antibody for flow cytometry analysis. b The resultant cells were also stained with APC-labeled anti-CD42 antibody for flow cytometry analysis. c Mature megakaryocytes were purified through a BSA density gradient and RNA was prepared for measuring mRNA expression of mouse Pithdl, and Runxl by quantitative RT-PCR. The Pithdl and Runxl expression were normalized to Gapdh and presented as relative expression. d Mouse

[10]. For instance, the RUNX1 knockin mice deleted for IRES element mimicked some features of RUNX1-deficient mice. These mice showed marked reduction, altered differentiation, increased apoptosis, and decreased cell cycle in fetal liver hematopoietic cells. What is more, fetal liver cells from these mice showed decreased repopulation ability. However, how IRES-dependent RUNX1 upregulation is activated in embryogenesis remains unknown. Dissecting the mechanism is very helpful to understand the function of RUNX1. In general, IRES elements are cisacting sequences that form secondary and tertiary RNA structures and recruit IRES tans-activating factors (ITAFs) fetal liver cells were transduced with control lentiviral vector (Ctrl) or PITHD1-downregulating (shRNA/m\#1, shRNA/m\#2) vector and cultured for megakaryocytes. The resultant cells were stained with PE-labeled anti-CD41 antibody for flow cytometry analysis. e The resultant cells were also stained with APC-labeled anti-CD42 antibody for flow cytometry analysis. f RNA was prepared from purified mature megakaryocytes for measuring mRNA expression of mouse Pithd1, and Runxl by quantitative RT-PCR. The Pithdl and Runxl expression were normalized to Gapdh and presented as relative expression. *Indicates significance $(p<0.05)$

and translation machinery to an internal position in the mRNA in contrast to the cap-dependent initiation mechanism [23, 24]. With the exception of one or more polypyrimidine tracts, neither primary sequence conservation nor overall structural similarity is detected in IRES elements, strongly suggesting that different strategies may be used to recruit the ribosomal subunits. In this study, we found that RUNX1 IRES was only active in K562 but not 293T cells (data not shown). Furthermore, PITHD1 significantly activates RUNX1 and ECMV IRES-mediated protein translation (Fig. 5d, e), suggesting PITHD1 may act as a novel ITAF. It is very interesting to further probe 
how RUNX1 IRES is regulated by PITHD1 in embryogenesis. Considering the critical function of RUNX1 in embryo hematopoiesis, PITHD1 may also participate in this process by modulating RUNX1 expression.

The effect of PITHD1 on blood cell differentiation appears not to be limited to megakaryocyte lineage. We found that PITHD1 overexpression also promoted erythrocyte differentiation induced by Ara-C or hemin in K562 cells evidenced by increased $\gamma$-globin expression (Figure S2). Considering its significant downregulation in AML, it is possible that PITHD1 deregulation may play a role in leukemogenesis of AML.

In a word, we have identified the novel function of an uncharacterized new gene PITHD1 that may promote leukemic megakaryocyte differentiation as well as normal megakaryopoiesis. PITHD1 may do so in part by acting as a novel ITAF to upregulate RUNX1. As far as we know, this is the first line of evidence showing that there exists cellular protein acting as an IRES trans-activating factor to regulate RUNX1 expression in IRES-dependent manner. Our findings shed light on understanding the mechanisms underlying megakaryopoiesis and leukemogenesis.

Acknowledgments The authors thank College of Life Sciences Flow Cytometry Core Facility for technical support. This work was supported by National Natural Science Foundation of China (31371481 to Z. Huang), Ph.D. Programs Foundation of Ministry of Education of China (Grant No. 20110141110016 to Z. Huang), and Program for New Century Excellent Talents in University (NCET) of Ministry of Education of China (Grant No. NCET-12-0422 to Z. Huang), the Chinese 111 project (Grant No. B06018 to H. Shu).

\section{References}

1. Wang ZY, Chen Z (2008) Acute promyelocytic leukemia: from highly fatal to highly curable. Blood 111(5):2505-2515

2. Chen SJ, Zhou GB, Zhang XW, Mao JH, de The H, Chen Z (2011) From an old remedy to a magic bullet: molecular mechanisms underlying the therapeutic effects of arsenic in fighting leukemia. Blood 117(24):6425-6437

3. Wen Q, Goldenson B, Silver SJ, Schenone M, Dancik V, Huang Z, Wang LZ, Lewis TA, An WF, Li X, Bray MA, Thiollier C, Diebold L, Gilles L, Vokes MS, Moore CB, Bliss-Moreau M, Verplank L, Tolliday NJ, Mishra R, Vemula S, Shi J, Wei L, Kapur R, Lopez CK, Gerby B, Ballerini P, Pflumio F, Gilliland DG, Goldberg L, Birger Y, Izraeli S, Gamis AS, Smith FO, Woods WG, Taub J, Scherer CA, Bradner JE, Goh BC, Mercher T, Carpenter AE, Gould RJ, Clemons PA, Carr SA, Root DE, Schreiber SL, Stern AM, Crispino JD (2012) Identification of regulators of polyploidization presents therapeutic targets for treatment of AMKL. Cell 150(3):575-589

4. Ichikawa M, Yoshimi A, Nakagawa M, Nishimoto N, WatanabeOkochi N, Kurokawa M (2013) A role for RUNX1 in hematopoiesis and myeloid leukemia. Int J Hematol 97(6):726-734

5. Elagib KE, Racke FK, Mogass M, Khetawat R, Delehanty LL, Goldfarb AN (2003) RUNX1 and GATA-1 coexpression and cooperation in megakaryocytic differentiation. Blood 101(11):4333-4341
6. Ichikawa M, Asai T, Saito T, Seo S, Yamazaki I, Yamagata T, Mitani K, Chiba S, Ogawa S, Kurokawa M, Hirai H (2004) AML1 is required for megakaryocytic maturation and lymphocytic differentiation, but not for maintenance of hematopoietic stem cells in adult hematopoiesis. Nat Med 10(3):299-304

7. Ichikawa M, Goyama S, Asai T, Kawazu M, Nakagawa M, Takeshita M, Chiba S, Ogawa S, Kurokawa M (2008) AML1/ Runx1 negatively regulates quiescent hematopoietic stem cells in adult hematopoiesis. J Immunol 180(7):4402-4408

8. Growney JD, Shigematsu H, Li Z, Lee BH, Adelsperger J, Rowan R, Curley DP, Kutok JL, Akashi K, Williams IR, Speck NA, Gilliland DG (2005) Loss of Runx1 perturbs adult hematopoiesis and is associated with a myeloproliferative phenotype. Blood 106(2):494-504

9. Ghozi MC, Bernstein Y, Negreanu V, Levanon D, Groner Y (1996) Expression of the human acute myeloid leukemia gene AML1 is regulated by two promoter regions. Proc Natl Acad Sci USA 93(5):1935-1940

10. Nagamachi A, Htun PW, Ma F, Miyazaki K, Yamasaki N, Kanno M, Inaba T, Honda Z, Okuda T, Oda H, Tsuji K, Honda H (2010) A 5' untranslated region containing the IRES element in the Runx1 gene is required for angiogenesis, hematopoiesis and leukemogenesis in a knock-in mouse model. Dev Biol 345(2):226-236

11. Pozner A, Goldenberg D, Negreanu V, Le SY, Elroy-Stein O, Levanon D, Groner Y (2000) Transcription-coupled translation control of AML1/RUNX1 is mediated by cap- and internal ribosome entry site-dependent mechanisms. Mol Cell Biol 20(7):2297-2307

12. Yi H, Xue L, Guo MX, Ma J, Zeng Y, Wang W, Cai JY, Hu HM, Shu HB, Shi YB, Li WX (2010) Gene expression atlas for human embryogenesis. Faseb J Off Publ Fed Am Soc Exp Biol 24(9):3341-3350

13. Xue L, Yi H, Huang Z, Shi YB, Li WX (2011) Global gene expression during the human organogenesis: from transcription profiles to function predictions. International $\mathrm{J}$ Biol Sci 7(7): $1068-1076$

14. Sun X, Lu B, Hu B, Xiao W, Li W, Huang Z (2014) Novel function of the chromosome 7 open reading frame 41 gene to promote leukemic megakaryocyte differentiation by modulating TPA-induced signaling. Blood Cancer J 4:e198

15. Muntean AG, Crispino JD (2005) Differential requirements for the activation domain and FOG-interaction surface of GATA-1 in megakaryocyte gene expression and development. Blood 106(4):1223-1231

16. Huang Z, Dore LC, Li Z, Orkin SH, Feng G, Lin S, Crispino JD (2009) GATA-2 reinforces megakaryocyte development in the absence of GATA-1. Mol Cell Biol 29(18):5168-5180

17. Jacquel A, Herrant M, Defamie V, Belhacene N, Colosetti P, Marchetti S, Legros L, Deckert M, Mari B, Cassuto JP, Hofman $\mathrm{P}$, Auberger P (2006) A survey of the signaling pathways involved in megakaryocytic differentiation of the human K562 leukemia cell line by molecular and c-DNA array analysis. Oncogene 25(5):781-794

18. Huang H, Yu M, Akie TE, Moran TB, Woo AJ, Tu N, Waldon Z, Lin YY, Steen H, Cantor AB (2009) Differentiation-dependent interactions between RUNX-1 and FLI-1 during megakaryocyte development. Mol Cell Biol 29(15):4103-4115

19. Tijssen MR, Cvejic A, Joshi A, Hannah RL, Ferreira R, Forrai A, Bellissimo DC, Oram SH, Smethurst PA, Wilson NK, Wang X, Ottersbach K, Stemple DL, Green AR, Ouwehand WH, Gottgens B (2011) Genome-wide analysis of simultaneous GATA1/2, RUNX1, FLI1, and SCL binding in megakaryocytes identifies hematopoietic regulators. Dev Cell 20(5):597-609

20. Hart A, Melet F, Grossfeld P, Chien K, Jones C, Tunnacliffe A, Favier R, Bernstein A (2000) Fli-1 is required for murine vascular 
and megakaryocytic development and is hemizygously deleted in patients with thrombocytopenia. Immunity 13(2):167-177

21. Satoh Y, Matsumura I, Tanaka H, Ezoe S, Fukushima K, Tokunaga M, Yasumi M, Shibayama H, Mizuki M, Era T, Okuda T, Kanakura Y (2008) AML1/RUNX1 works as a negative regulator of c-Mpl in hematopoietic stem cells. J Biol Chem 283(44):30045-30056

22. Lee S, Kim SM, Lee RT (2013) Thioredoxin and thioredoxin target proteins: from molecular mechanisms to functional significance. Antioxid Redox Signal 18(10):1165-1207
23. Pacheco A, Martinez-Salas E (2010) Insights into the biology of IRES elements through ribo proteomic approaches. J Biomed Biotechnol 2010:458927

24. Thompson SR (2012) Tricks an IRES uses to enslave ribosomes. Trends Microbiol 20(11):558-566 